Alfarama Journal of Basic \& Applied Sciences

Faculty of Science Port Said University https://ajbas.journals.ekb.eg

ajbas@sci.psu.edu.eg

http://sci.psu.edu.eg/en/

DOI: $10.21608 / A J B A S .2020 .24150 .1011$

\title{
Physical Parameters Which Help in Diagnosis of Urinary Tract Obstruction by Renal Scintigraphy.
}

\author{
Hoda Abdelraouf ${ }^{1, *}$, Hossam M.Gad ${ }^{1}$, Mossad El-Metwally ${ }^{2}$ \\ ${ }^{1}$ Radiology department, Urology and Nephrology Center, Mansoura University, Egypt. \\ ${ }^{2}$ Physics Department, Faculty of Science, Port-Said University, Port-Said, Egypt. \\ *Corresponding author:Hoda dody2002@yahoo.com
}

\begin{abstract}
Background: Diuresis renography is the most commonly test used tool to investigate the urinary tract dilatation, which differentiate between obstructed and non-obstructed systems and evaluate the glomerular filtration rate (GFR). This test is widely accepted because of its convenience and noninvasiveness. Prompt clearance of the radiopharmaceutical from the renal pelvis with a $T_{1 / 2}$ of less than $10 \mathrm{~min}$ is a normal response, values between 10 and $20 \mathrm{~min}$ are considered equivocal results, and a $\mathrm{T} 1 / 2$ greater than 20 min suggests obstruction. Objective: This study aims to assess the optimal timing of furosemide injection in diuretic renography in order to obtain the most accurate diagnosis.
\end{abstract}

Materials and methods: This study included 300 renal units (RU) were examined at Urology and Nephrology Center- Mansoura University from 2016 to 2019 by F+10 min diuretic renography protocol. Results: One hundred and seventeen (RU) of 300 (RU) gave no response to diuresis and were diagnosed as obstructive kidneys. One hundred and twenty one (RU) revealed the excretion of kidneys were delayed but improved diuresis response and were diagnosed as non-obstructive kidneys, The remaining sixty two (RU) showed equivocal response to diuresis after diuretic were done and underwent F-15 min protocol of diuretic renography. Twenty (RU) were proved to be non-obstructed, forty two (RU) showed complete obstruction. Comparing the results of F+10min protocol with F-15 min protocol for the $62(\mathrm{RU})$ with equivocal response $42(\mathrm{RU})$ revealed as an obstructive kidneys by using F-15min protocol with mean $\pm \mathrm{SD} \mathrm{T}^{1} / 2$ values $36.64 \mathrm{~min} \pm 14.70 \mathrm{~min}$ which was $15.19 \mathrm{~min} \pm 2.70$ by $\mathrm{F}+10 \mathrm{~min}(\mathrm{p}=.000)$. The other twenty $(\mathrm{RU})$ revealed as nonobstructive kidneys and have mean $\pm \mathrm{SD} \mathrm{T} 1 \frac{1}{2}$ values $9.83 \mathrm{~min} \pm 3.33 \mathrm{~min}$ by using $\mathrm{F}-15 \mathrm{~min}$ protocol which was $14.43 \pm 2.96 \mathrm{~min}$ by using $\mathrm{F}+10 \mathrm{~min}(\mathrm{p}=.0 .000)$. A $\mathrm{p}$ value $<.05$ was considered significant.

Conclusion: F-15 min diuretic renography protocol can be considered as the most sensitive and gold standard method for making a distinction between urinary obstruction and non-obstruction.

\section{Keywords:}

diuresis renography, equivocal, kidney obstruction, furosemide, DTPA. 


\section{INTRODUCTION}

Nuclear medicine imaging includes the use of radio-pharmaceuticals which are generally used to detect and assess disease in the human body. The radiopharmaceutical is administered, inhaled or being injected intravenously, the choice of pharmaceutical and the method of administration depends on the disease being investigated [1]. These devices have a main role in diagnosis and follow up of various renal diseases and characterized by highly sensitivity that initiate early detection of disease. There is a recent technical advances in (CT), (MRI), and (US) but nuclear medicine (NM) techniques maintain (gold standard) status in the diagnosis of upper urinary tract obstruction [2].

Diuresis renography (DR) is a non-invasive, widely available method that used for assessment function of the kidney (GFR) and testing the urodynamics in a single procedure. This non-invasive method is depending on a high endogenous urine flow rate which the furosemide being administered; this test always been checked by using radiopharmaceutical washout from the upper urinary tract collecting system [3].

In 1978, O'Reilly et al [4-5] introduced the first procedure: a dosage of $40 \mathrm{mg}$ of furosemide was given 20 minutes after ${ }^{99 \mathrm{~m}} \mathrm{Tc}-\mathrm{DTPA}$ was injected $\left({ }^{99 \mathrm{~m}} \mathrm{Tc}\right.$-DTPA subsequently replaced by ${ }^{99 \mathrm{~m}} \mathrm{Tc}-\mathrm{MAG} 3$ which has the highly rate of extraction), and this method called F+20 minutes protocol which the dynamic acquisition continued for another 20 minutes after the furosemide was administrated. Hydronephrosis (HDN) in adults is a common presentation and may be lead to congenital uretero-pelvic junction obstruction [6].

A dilation of the renal pelvis, ureter and calyces caused by the obstruction to free urine flow from the kidney, leading to an advanced atrophy of the renal cortex. It can be the result of an anatomical or functional mechanism that obstructs the urine flow, which can occur from the kidneys to the urethral meatus anywhere [7].

Diuresis renography (DR) used to diagnoses a differentiation renal collecting system dilation, it has the benefit to asses the relative function of the renal, determine the washout of tracer's diuretic-promoted and to provide the possibility for using it to infants with low radiation exposure [8].

Obstruction classified according to the site, degree and duration to acute or chronic obstruction which occurs anywhere in the urinary tract and includes intra-renal causes (casts, crystals) and extra-renal causes [9]. O'Reilly et al., [4] studied the equivocal urinary tract obstruction patient's; he found that using diuretic renogram was preferred to evaluate the equivocal urinary tract obstruction of the patient. Diuretic renograhy was described by using 99mTc-DTPA as a renal radiopharmaceutical which was the only radiopharmaceutical purely filtered by the glomerulus and used to measure glomerular filtration rate (GFR) [10].

Diuretic response is a quantitative rate of washout by determining the $T_{1 / 2}, T_{1 / 2}$ refer to the time talked for the activity in the kidney to decrease to 50 percent of its maximum value). When $\mathrm{T}_{1 / 2}<10 \mathrm{~min}$ a complete clearance of the ${ }^{99 \mathrm{~m}}$ TC- DTPA from the renal pelvis, this refer to there is no obstruction and the kidney is normal washout. When the values of $\mathrm{T}_{1 / 2}$ between 10 and $20 \mathrm{~min}$ this refer to the kidney has equivocal response for diuresis. When $\mathrm{T}_{1 / 2}>20$ min was diagnosed as obstructed kidney [6].

There are three items which Diuresis renography based on:

1. The patient should be having a good state of hydration: A $500 \mathrm{ml}$ water or juice was needed to obtain a good oral hydration in all patients. 
2. The optimal time to inject the furosemide, It is typically a dosage in adults ( $40 \mathrm{mg}), 0.5$ $\mathrm{mg} / \mathrm{kg}$ in children and $1 \mathrm{mg} / \mathrm{kg}$ in infants. Some protocols introduce the administration time of furosemide between ( 20-30 min) after tracer injection known as F+20 min protocol[11]. However, as this may result in a significant number of equivocal responses, a protocol was introduced to decrease the number of equivocal response known as (F-15 min protocol) which furosemide was injected $15 \mathrm{~min}$ before radiopharmaceutical injection. Therefore, administering the F-15 min protocol by injection of radiopharmaceutical 15 min before examination to obtain a maximum diuresis of the kidney throughout the scan [12].

3. The role of the bladder, the bladder should be emptied before starting the study. [13]. This can be found that increased the pressure of pelvic pressure during bladder filling leading to a reduction in the drainage of the pelvicaliceal system, which a false positive results will be occurred [12].

The F+10 protocol leads to equivocal results of $15 \%$ to $17 \%$ and therefore, the method of the F-15 has been administrated to reduce this rate to $3 \%$ [15]. The use of the F-15 method results in a longer study period and bladder overdistension that may cause a maximum diuresis of the kidney which reduce the rate of equivocal response. This work aims to determine the optimum timing of furosemide injection in diuretic renography to get the most accurate diagnosis in the equivocal cases by administrating two comparative protocols (F+10 min protocol, F-15 min protocol) for differentiate between obstructed and non-obstructed cases.

\section{EXPERIMENTAL}

\subsection{Subjects}

One hundred and fifty patients with 300 renal units (RU) were prospectively evaluated during 2016-2019 in the Urology and Nephrology Center at Mansoura University with suspected urinary obstruction. All cases administrated by $\mathrm{F}+10$ diuretic renography protocol, $117(\mathrm{RU})$ have mean $\mathrm{T}^{1} / 2$ value more than $20 \mathrm{~min}$ and diagnosed as obstructed kidneys and the other 121 (RU) had mean $\mathrm{T}^{1} / 2$ value less than 10min and diagnosed as non-obstructed kidneys. The remaining $62(\mathrm{RU})$ have mean $\mathrm{T}^{1 / 2} / 2$ value between $10 \mathrm{~min}$ to $20 \mathrm{~min}$ with indeterminate diagnosis (equivocal results), this remaining (RU) which showed equivocal response to diuresis were underwent to F-15 min diuretic renography.

\subsection{Apparatus and methods}

Renography procedures

A. Patient Preparation: The patient was checked about of information, appropriate weight and height and laboratory tests (e.g. Serum Creatinine and urea levels). A $500 \mathrm{ml}$ drink ( e.g, water, orange juice) was given 15 minutes before the examination. The patient was asked to void his bladder before the test.

B. Patient dosage: The radiopharmaceutical was ${ }^{99 \mathrm{~m}}$ Tc- DTPA, the dosage of ${ }^{99 \mathrm{~m}}$ Tc- DTPA adjusted according to body weight, the adult dose at the range $111-259 \mathrm{MBq}(3-7 \mathrm{mCi}) 0.05 \mathrm{mci} / \mathrm{kg}$. The furosemide dose was $0.5 \mathrm{mg} / \mathrm{kg}$ body weight.

C. Image preparation and Data acquisition: All patients were examined on Gamma Camera (bright view, Philips medical system company, City: United states of America ). The collimator was low energy general purposes, the photo-peak was selected at $140 \mathrm{keV}$, the total frames of the study phases are 76 frames during all study (20 min).

D. Patient Positioning: The patients were positioned prior to the injection in supine position. The field of view was verified with a marker prior to injection, both kidneys, ureters and bladder were included.

The two protocols of $\mathrm{F}+10 \mathrm{~min}$ and F-15min diuretic renography were performed as follows:

F + 10 Diuretic Renography protocol 
The patient at the state of good hydration (500ml water) before the study. The dose of ${ }^{99 \mathrm{~m}} \mathrm{Tc}$ DTPA was injected intravenously in the patient as being a bolus and then, the computer system was acquired data in frame mode. At ten (10) min, a furosemide dose of $0.5 \mathrm{mg} / \mathrm{kg}$ body weight was injected. the total time of acquisition was $20 \mathrm{~min}$.

\section{F -15 Diuretic Renography protocol}

After 2 days the same patient which had equivocal result for diuresis, the patient had a good condition of hydration and that there are no clinical reasons for diuresis. The patient was Injected furosemide at a dose $0.5 \mathrm{mg} / \mathrm{kg}$ body weight before $15 \mathrm{~min}$ of the study. The radiopharmaceutical was injected as a routine renogram and was continued until $20 \mathrm{~min}$. The total time of acquisition is 35 minutes.

\subsection{Statistical analysis}

The result of the curve pattern, drainage half time $\left(\mathrm{T}_{1 / 2}\right)$ and split renal function $(\%)$ in the two protocols $\mathrm{F}+10, \mathrm{~F}-15)$ were recorded and compared by the paired t test using the SPSS software (Statistical package for the social sciences, version 20). $\boldsymbol{P}<0.05$ was considered statistically significant.

\section{RESULTS}

This study included 150 patient with 300 renal units (RU) their ages ranged from 45 day to 70 year with mean \pm SD (27.94 \pm 21.41$)$. This patients were examined by the F+10min diuretic renography protocol, 117 (RU) had mean $\mathrm{T}^{1} \frac{1}{2}$ value more than 20min and diagnosed as obstructed kidneys (Fig 1) and the other 121 (RU) had mean $\mathrm{T}^{1} / 2$ value less than 10min and diagnosed as non-obstructed, well-functioning kidneys (Fig 2). The remaining 62 (RU) had mean $\mathrm{T}^{1 / 2}$ value between $10 \mathrm{~min}$ to $20 \mathrm{~min}$ with indeterminate diagnosis (equivocal results) (Fig 3).

After administrating F-15 min protocol for equivocal cases, the overall results indicated that obstruction was found in 159 (RU) out of 300 (RU) with mean \pm SD T1/2 values $37.42 \mathrm{~min} \pm 13.88 \mathrm{~min}$ and the mean kidney split function was $35.08 \% \pm 13.07 \%$. One hundred and forty one (RU) out of 300 (RU) were nonobstructed with mean $\pm \mathrm{SD} \mathrm{T}^{1} / 2$ values $7.94 \mathrm{~min} \pm 2.31 \mathrm{~min}$ and their mean kidney split function was $61.79 \% \pm 11.63 \%$.

Comparing the results of F+10min protocol with F-15 min protocol for the 62 (RU) with equivocal response 42 (RU) were proved to be obstructed by F-15min study with mean \pm SD T $1 / 2$ values $36.64 \mathrm{~min} \pm 14.70 \mathrm{~min}$ which was $15.19 \min \pm 2.70$ by $\mathrm{F}+10 \mathrm{~min}(\mathrm{p}=.000)$, which illustrated in $\mathrm{Fig}(4)$, the kidney split function mean \pm SD values $39.25 \% \pm 11.8 \%$ by F- $15 \mathrm{~min}$, which was $39.72 \% \pm 12.03 \%$ by $\mathrm{F}+10 \mathrm{~min}(\mathrm{p}=0.193)$. The other twenty (RU) were proved to be non-obstructed with mean \pm SD T $1 / 2$ values $9.83 \min \pm 3.33$ min in F- 15 min protocol which was $14.43 \pm 2.96 \mathrm{~min}$ in $\mathrm{F}+10 \mathrm{~min}(\mathrm{p}=0.000)$ which illustrated in Fig(5), the kidney split function mean \pm SD values $56.41 \% \pm 10.79 \%$ by F-15min, which was $56.49 \% \pm 10.23 \%$ by $\mathrm{F}+10 \mathrm{~min}$ $(\mathrm{p}=0.859)$. A p value less than .05 was considered significant.

\section{DISCUSSION}

Some radiologists explain the study by visually analyzing the washout curves such as cases of obstructed kidney and non-obstructed kidney, but some prefer analyzing the washout curves time for $\mathrm{T}_{1 / 2}$ (the activity in the collecting system to fall to $50 \%$ of its original value of the radiopharmaceutical).

Normal $T_{1 / 2}$ values depend upon the radiopharmaceutical, the delay between administering the radiopharmaceutical and administering furosemide, the method of hydration, the dose of furosemide, and the interval used to make the measurement. Prompt clearance of the radiopharmaceutical from the renal pelvis with a $\mathrm{T}_{1 / 2}$ of less than $10 \mathrm{~min}$ is a normal response, values between 10 and $20 \mathrm{~min}$ are considered equivocal results, and a $\mathrm{T} 1 / 2$ greater than 20 min suggests obstruction. 
In F-15 renography, the kidney takes enough time to deal with the furosemide to reach the maximum effect. This method helps in differentiating between kidney obstruction and non-obstruction.

In our study Sixty two (RU) with equivocal result in the F+10 protocol, 42 (RU) had an obstructive pattern in the F-15 with half time or $\mathrm{T}_{1 / 2}$ equal $36.64 \mathrm{~min}$ and had the value of $\mathrm{F}+10 \mathrm{~min}$ protocol equal $15.19 \mathrm{~min}$ ( $\mathrm{p}=.000$ ) which mean there is an increase in $\mathrm{T}_{1 / 2}$ when the diagnosis is obstructed pattern $\mathrm{T}_{1 / 2}>20$ so, there is a significant change in the value of $\mathrm{T}_{1 / 2}$ after F-15min protocol. The other twenty (RU) with equivocal pattern in the $\mathrm{F}+10$ protocol which proved to be non-obstructed, the value of $\mathrm{T}_{1 / 2}$ equal $14.43 \mathrm{~min}$, after $\mathrm{F}$ 15 the value of $\mathrm{T}_{1 / 2}$ equal $9.83 \mathrm{~min}\left(\mathrm{p}=.000\right.$ ), this mean that there is a decrease in $\mathrm{T}_{1 / 2}$ when the diagnosis is non- obstructed pattern $\mathrm{T}_{1 / 2}<10$.

In equivocal cases, some recommend repeating the study with the " $F$ minus 15 renogram" the furosemide is then given at 15 min before the radiopharmaceutical, so the patient will be in a good state of maximum diuresis at the time when the radiopharmaceutical is administered.

Kumar and Hanuwant studied on 51 adult patients of primary hydronephrosis. Each patient underwent both F+20 and F-15 diuretic Technetium-99m diethylenetriaminepentacetate $\left({ }^{99 m}\right.$ Tc-DTPA) renography protocol studies. They are found that A total $60 \mathrm{RU}$ with HDN (42 patients with unilateral and 9 patients with bilateral HDN) were included in this study. The equivocal results were significantly lower in F-15 protocol (1/60) than F+20 protocol (24/60), So the widely practiced F+20 protocol has very high incidence of inconclusive results ranging from 10 to $40 \%$ [2].

Similarly, in our study 62 (RU) 20.6\% were equivocal in F+10 protocol. The F-15 protocol conclusively categorized 20 (RU) as nonobstructed, 42 (RU) as a obstructed, so there are a clear reduction in the number of equivocal cases and this mean that F-15 min protocol is the gold stander method for differentiation between obstruction and non-obstruction cases.

Babu and Venkatsubramaniam; studied on 148 diuretic renograms in infants and children to evaluate unilateral Grade 3-4 HDN. The number of interrupted studies was significantly less in F+0 compared with $\mathrm{F}-15$ and $\mathrm{F}+20$. The $\mathrm{F}+0$ and $\mathrm{F}-15$ protocols are superior to the $\mathrm{F}+20$ protocol in reducing the number of equivocal curves [14]. Similarly in our study the number of equivocal response reduced by using F-15 min protocol.

Taghavi and Ariana; studied on 21 patient with pyelocaliceal system dilatation used F+20 and F-15 Methods for Differentiation of Upper Urinary Tract Dilatation From Obstruction. The patients underwent diuresis renography using the $\mathrm{F}+20$ and $\mathrm{F}-15$ protocols. The overall results, obstruction was found in 16 out of 21 patients $(76.2 \%)$ by the $\mathrm{F}-15$ protocol, while it was found in $11(52.4 \%)$ by the $\mathrm{F}+20$ protocol $(\mathrm{P}=$ $0.01)[15]$.

In our study the patients underwent diuresis renography using the F+10 and F-15 protocol. The overall results, obstruction found in 159 (53\%) by the F-15 protocol, while it was found in $117(39 \%)$ by F+10 protocol $(\mathrm{P}=0.000)$.

Foda et al [16]. studied on a 72 children were randomly assigned to 1 of 2 standardized diuresis renography protocols. The F-15 scan showed 7 times more obstruction than the F+20 scan on the investigated side, and this difference was statistically significant.

In our study there was a statistical significant of the drainage time $\left(\mathrm{T}_{112}\right)$ between $\mathrm{F}+10$ min protocol and $\mathrm{F}$ 15 min protocol, the latter is longer and $(\mathrm{P}=0.000)$. 

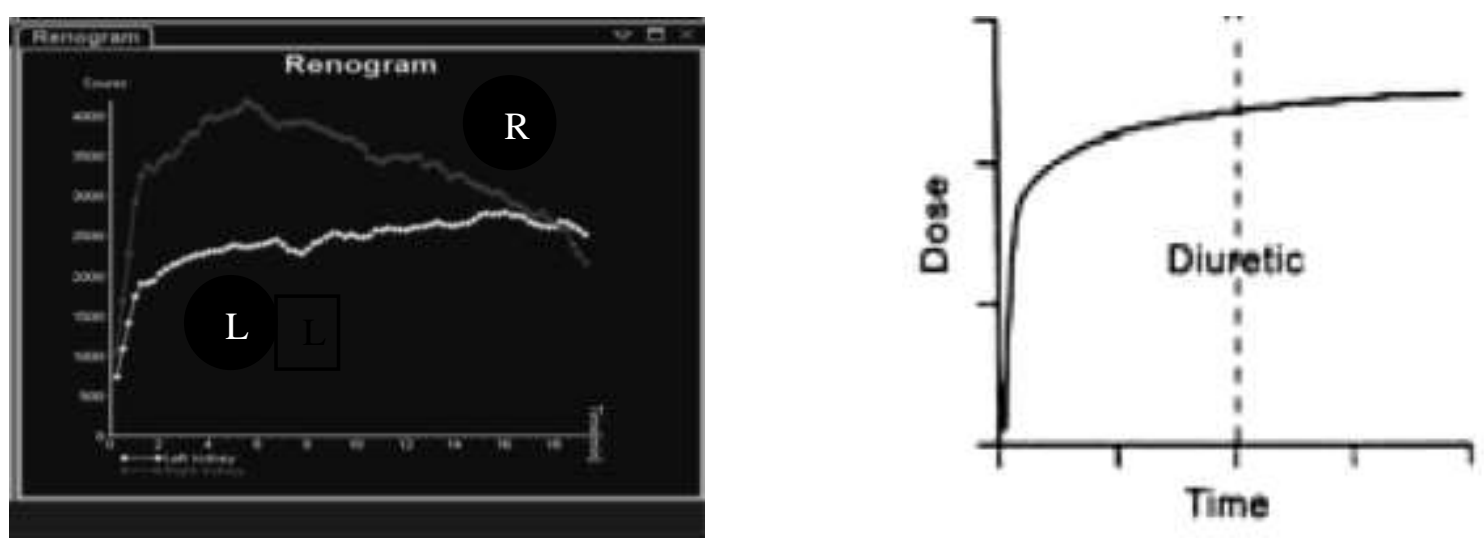

Figure (1): A patient with suspected urinary obstruction underwent F+10min the renograme curve indicated that there is an obstruction in the left kidney, Non obstructed right kidney.
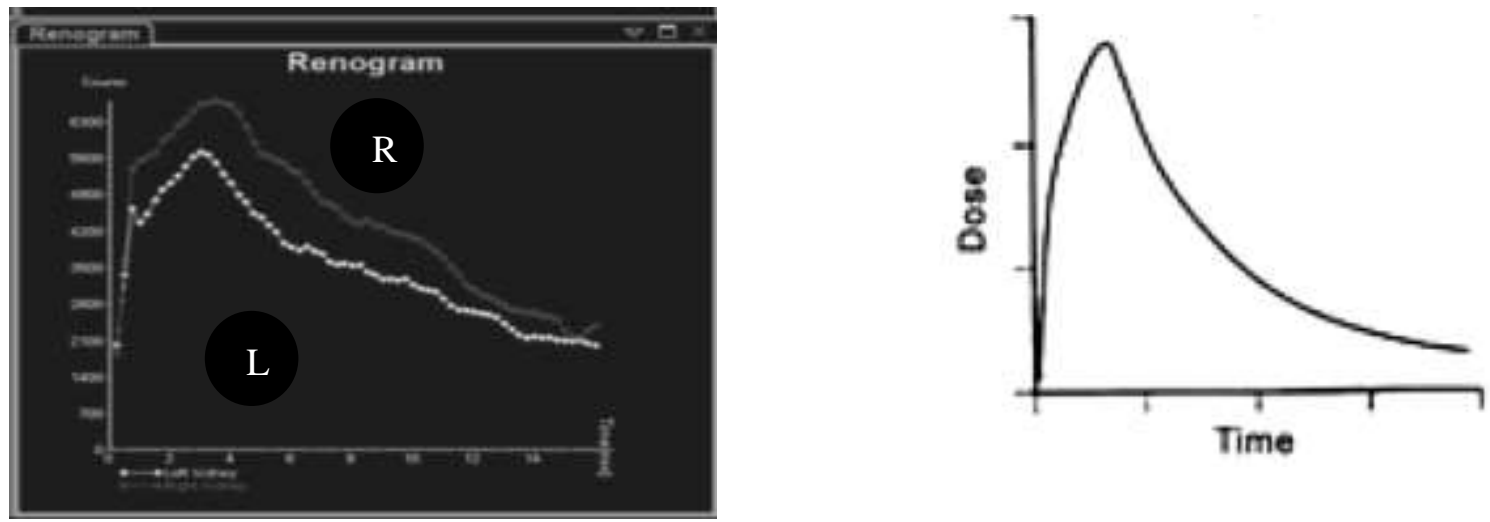

Figure (2): A patient with suspected urinary obstruction underwent F+10min the renograme curve indicated that there is there is no obstruction in both kidneys.
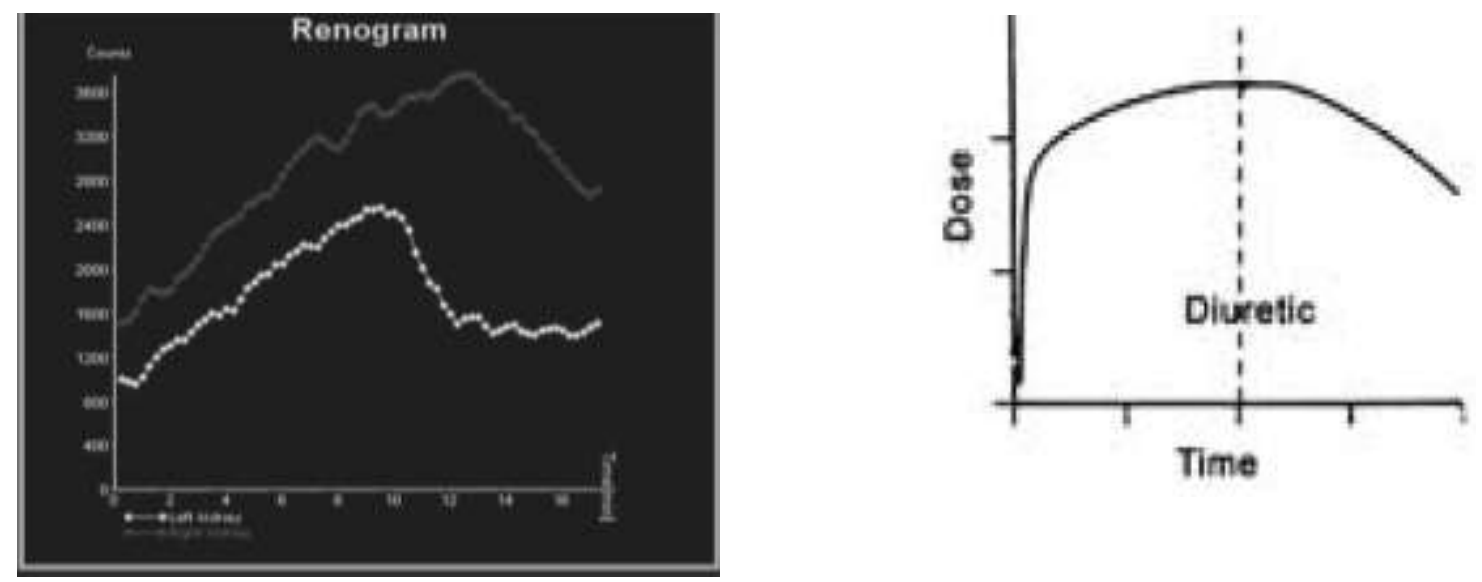

Figure (3): A patient with suspected urinary obstruction underwent F+10min the renograme curve indicated that there is an equivocal response to diuresis at the left kidney. F-15 min is recommended for further assessment of the left kidney, Well function non obstructed right kidney. 


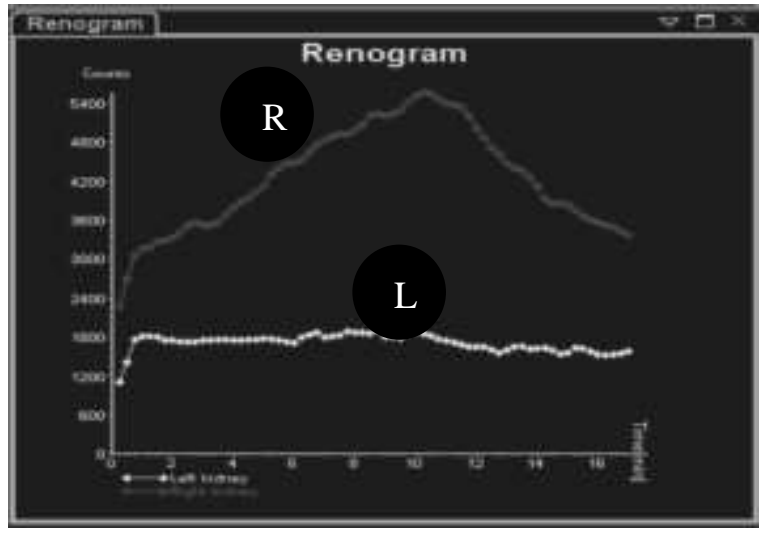

Figure (4): In the same patient after time by applying F-15 min protocol, furosemide was injected I.V 15 minutes before injection of Tc$99 \mathrm{~m}$ DTPA. The left kidney showed reduced perfusion \&tracer uptake and delayed excretion without improvement in response to diuresis (obstructed kidney).

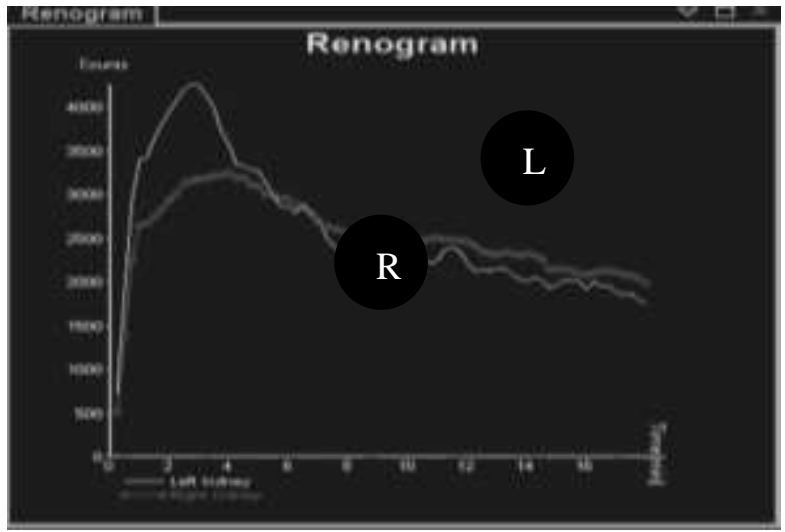

Figure (5): A patient with non-obstructive right kidney which underwent to F-15 min protocol. The renogram curve indicated that the right kidney is non-obstructed afterF-15 min protocol administration.

\section{CONCLUSION}

In our study, F-15 diuresis renogram protocol was associated with significantly less equivocal results than F+10 protocol. Moreover, F-15 protocol allowed explanation the cases of equivocal results of F+10 protocol. Therefore, F-15 diuretic renogram protocol is the gold standard and sensitive radiological method for diagnosis of urinary obstruction with which $\mathrm{T}_{1 / 2}$ time is calculated correctly to be adjuvant physical parameter for confirming diagnosis.

\section{REFERENCES}

[1] Staelens, S.; Buvat, I. Monte Carlo simulations in nuclear medicine imaging. Advances in Biomedical Engineering. Elsevier., 177-209, 2009.

[2] Cosgriff, P. S. The urinary tract. Practical nuclear medicine., Springer, London., 205-229, 2005.

[3] Taylor, A. T. Radionuclides in nephrourology, Part 2: pitfalls and diagnostic applications. Journal of Nuclear Medicine., 55.5, 786-798, 2014.

[4] O'reilly, P. H.; Testa, H. J.; Lawson, R. S.; Farrar, D. J.; Edwards, E. C. Diuresis renography in equivocal urinary tract obstruction. British Journal of Urology., 50.2, 76-80, 1978.

[5] Tartaglione, G.; D’Addessi, A.; De Waure, C.; Pagan, M.; Raccioppi, M.; Sacco, E.; Al-Nahhas, A. 99mTc-MAG3 Diuretic Renography in Diagnosis of Obstructive Nephropathy in Adults: A Comparison Between F- 15 and a new Procedure F+ 10 (sp) in Seated Position. Clinical nuclear medicine., 38.6, 432-436, 2013.

[6] Kumar, M. T.; Hanuwant, S. Comparison of the F+ 20 and F-15 diuresis technetium-99m diethylenetriaminepentacetate renography protocols for diagnosis of ureteropelvic junction obstruction in adult patients with hydronephrosis. Indian journal of nuclear medicine: IJNM: the official journal of the Society of Nuclear Medicine; India 33.1, 39, 2018.

[7] Iqbal, S.; Raiz, I.; Faiz, I. Bilateral hydroureteronephrosis with a hypertrophied, trabeculated urinary bladder. The Malaysian journal of medical sciences., MJMS 24.2, 106, 2017. 
[8] Tartaglione, G.; Townsend, D. M.; Bassi, P. F.; Bolton, R. C. D.; Giammarile, F.; Rubello, D. Diuresis renography in equivocal urinary tract obstruction. A historical perspective. Biomedicine \& Pharmacotherapy., 116, 10898, 2019.

[9] Floege, J.; Johnson, R. J.; Feehally, J. Comprehensive Clinical Nephrology E-Book: Expert Consult-Online and Print, Elsevier Health Sciences., 2010.

[10] Taylor, A. T. Radionuclides in nephrourology, part 1: radiopharmaceuticals, quality control, and quantitative indices." Journal of Nuclear Medicine., 55.4, 608-615, 2014.

[11] Jamar, F.; Barone, R. Renal imaging. Diagnostic Nuclear Medicine., Springer, Berlin, Heidelberg, 83-100, 2006.

[12] Baert, A. L. Diagnostic nuclear medicine. Springer Science \& Business Media, 2013.

[13] Jones, D. A.; Lupton, E. W.; George, N. J. R. Effect of bladder filling on upper tract urodynamics in man. British journal of urology., 65.5, 492-496, 1990.

[14] Babu, R., Venkatsubramaniam, D.; Venkatachalapathy, E. "F+ 0 diuretic protocol is superior to F15 and $\mathrm{F}+20$ for nuclear renogram in children." Indian journal of urology: IJU: journal of the Urological Society of India.; 31.3, 245, 2015.

[15] Taghavi, R.; Ariana, K.; Arab, D. "Diuresis renography for differentiation of upper urinary tract dilatation from obstruction: F+ 20 and F-15 methods." Urology journal., 4.1, 36-40, 2009.

[16] Foda, M. M. R.; Gatfield, C. T.; Matzinger, M.; Briggs, V.; Wells, G.; Walker, S.; Schillinger, J. F. "A prospective randomized trial comparing 2 diuresis renography techniques for evaluation of suspected upper urinary tract obstruction in children." The Journal of urology., 159.5, 16911693,1998. 